\title{
Comparative Genomics Study of Candidatus Carsonella Ruddii; an Endosymbiont of Economically Important Psyllids
}

\author{
Shakhinur Islam Mondal ${ }^{1 *}$, Elma Akter ${ }^{1}$, Arzuba Akter ${ }^{2 *}$, Md Tahsin Khan ${ }^{1}$, and Nurnabi Azad Jewel ${ }^{1}$ \\ ${ }^{1}$ Genetic Engineering and Biotechnology Department, Shahjalal University of Science and Technology, Sylhet, Bangladesh \\ ${ }^{2}$ Biochemistry and Molecular Biology Department, Shahjalal University of Science and Technology, Sylhet, Bangladesh
}

\begin{abstract}
Candidatus Carsonella ruddii is an endosymbiont that resides in specialized cells within the body cavity of plant sap-feeding insects called psyllids. The establishment of symbiotic associations is considered one of the key factors for the evolutionary success of psyllids, as it may have helped them adapt to imbalanced food resources like plant sap. Although $C$. ruddii is defined as a psyllid primary symbiont, the genes for some essential amino acid pathways are absent. Complete genome sequences of several $C$. ruddii strains have been published. However, in-depth intra-species comparison of $C$. ruddii strains has not yet been done. This study therefore aimed to perform a comparative genome analysis of six $C$. ruddii strains, allowing the interrogation of phylogenetic group, functional category of genes, and biosynthetic pathway analysis. Accordingly, overall genome size, number of genes, and GC content of $C$. ruddii strains were reduced. Phylogenetic analysis based on the whole genome proteomes of 30 related bacterial strains revealed that the six $C$. ruddii strains form a cluster in same clade. Biosynthetic pathway analysis showed that complete sets of genes for biosynthesis of essential amino acids, except tryptophan, are absent in six $C$. ruddii strains. All genes for tryptophan biosynthesis are present in three $C$. ruddii strains (BC, BT, and YCCR). It is likely that the host may depend on a secondary symbiont to complement its deficient diet. Overall, it is therefore possible that $C$. ruddii is being driven to extinction and replacement by new symbionts.
\end{abstract}

Keywords: Carsonella, endosymbiont, comparative genomics, pathway analysis

\section{Introduction}

Insects in the family of psyllidae (phylum Arthropoda, class Insecta) established a persistent intimate mutualistic association with the intracellular endosymbionts. Commonly these intracellular endosymbionts possess greatly reduced gene poor genome, which also influences

\footnotetext{
*Corresponding author

S. I. M.

Tel: +880821-714479, Fax: +880-821-715257

E-mail: shakhin200-gen@sust.edu

A. A.

E-mail: arzuba-bmb@sust.edu

(C) 2020, The Korean Society for Microbiology and Biotechnology
}

much fundamental biological process on their insect's host. Insects of these families can easily penetrate the plants tissue by flexible mouthparts sucking the plants phloem sap which is rich in sugars but poor in nitrogenated compound especially essential amino acids and this mood of feeding can highly influence for the transmission virus and other infectious agents and thus cause vectors of pathogens for agriculturally important plants [1-3]. Psyllid is economically important insects of these psyllidae family as they are responsible for various diseases in plants such as 'Tomato Vein-Greening' and 'Tomato Psyllid Yellows', in tomatoes [4] and 'Zebra Chip Disease' in potatoes [5] and citrus disease known as 'Huanglongbing' (HLB) or 'Greening Disease' [6] and 
cause a great loss in crop productions and yields. A common feature of organisms/insects that live on diets containing an excess of one class of compounds and a deficiency in essential nutrients could be fulfilled by the presence of intracellular symbionts (endosymbionts) that may provide the missing essential nutrients [7, 8]. Since psyllid has to survive under nutrients deficientunbalanced diets and stressful conditions, they have developed strong symbiotic relationships with their microbiome [9].

The microbiome of insects comprehends in a consortium of microbial communities colonizing the external and internal body structures, which are involved in a wide range of important functions such as colonization and resistance to pathogens, nutrient recycling and production, providing essential nutrients into the host [10]. The symbiotic relationship between unicellular and multicellular organisms has significantly contributed to their evolution and also influences the biological process for both insects and bacteria.

In case of psyllids and other plant-sap sucking Hemiptera, the endosymbiotic microorganism are located in specific organ like structure called bacteriomes- a specialized cell known as bacteriocyte within the insects body cavity [11]. The bacteria insects endosymbiotic relationship is maintained across generations through transovarial transmission where part of the free symbionts or intact bacteriocytes present in the maternal bacteriome migrate to the ovaries, and depending on the hosts they are deposited at various stages of oogenesis in the ovaries [12, 13]. Psyllids maternally harbor the endosymbiont Candidatus Carsonella ruddii (C. ruddii), a prokaryotic endosymbiont which maintains one of the smallest and most AT-rich bacterial genomes ever identified and has lost many genes that are thought to be essential for providing nutrients requirement of its host $[9,13]$. The extremely reduced genome with $160 \mathrm{~kb}$ in size containing a few genes involved in DNA repair, energy metabolism and cell envelope synthesis [13, 14]. The ability to perform most essential functions for a cell to be considered alive is heavily impaired in C. ruddii by the lack of genes involved in DNA replication, transcription and translation. Furthermore, in some cases, the shortening of genes causes the loss of essential domains and functional residues needed to fulfill such vital functions. In addition, at least half of the pathways towards the biosynthesis of essential amino acids, its proposed symbiotic functions, are completely or partially lost [15].

Here we present the comparative genome analysis of six C. ruddii strains isolated from six different psyllid host to identify the variation of genomic sequence and features, gene composition, shared genes and unique genes and gene lost and gain, and also the metabolic and biosynthetic pathway analysis in this small genome in order to gain a new acumens on the physiological role of this endosymbionts. So the in-depth genome comparisons of $C$. ruddii strains provide the insights into the evolutionary stability of the endosymbionts genomic and as well as metabolic architecture, which help to identify the novel control strategies by targeting this endosymbiont for psyllids pests.

\section{Materials and Methods}

\section{Sequence retrieval}

Nucleotide sequences of whole genomes of six C. ruddii strains namely $C$. ruddii BC (Accession number CP019943.1), C. ruddii BT (Accession number CP02479 8.1), C. ruddii YCCR (Accession number CP012411.1), C. ruddii CE (Accession number CP003541.1), C. ruddii HT (Accession number CP003544.1), C. ruddii PV (Accession number AP009180.1) were retrieved from NCBI genome database (https://www.ncbi.nlm.nih.gov/ genome). The information about genomic features and genes of six strains were curated genome viewer Artemis (https://www.sanger.ac.uk/science/tools/artemis).

\section{Whole genome comparative visualization}

BLAST Ring Image Generator (BRIG) [16] was used to show a genome wide visualization of coding sequences identity among different $C$. ruddii strains.

\section{Phylogenetic tree construction}

The CVTree3 is the alignment and parameter free whole genome based web server approach used for phylogenetic tree construction. This web server based method relies on the oligopeptide content (K-tuple length) of conserved proteins to deduce evolutionary relatedness which is using the neighbour-joining method based on dissimilarity matrix [17]. Trees constructed by the web server are not subjected to statistical re-sampling (boot- 
strap or jackknife analyses) because the underlying method emphasizes on the "objective" correctness of phylogeny with respect to taxonomy. The protein sequences of 30 different genomes/strains including the six $C$. ruddii strains used in this study were downloaded from NCBI genome database (https://www.ncbi.nlm.nih.gov/ genome) and saved as multifasta file with the extension .faa. In CVTree3 web server, the multifasta files of subjected sequence were uploaded. The outcome result was analysed by selecting K-tuple length options with a range from 5 to 6 as the best K-values for bacteria was shown to be 5-6 [17]. The best-supported neighbor-joining tree was visualised using FigTree v1.3.1 (http:// tree.bio.ed.ac.uk/software/figtree/).

\section{Functional analysis of predicted coding genes}

Protein sequences of the six $C$. ruddii strains were analysed with KEGG (Kyoto Encyclopaedia of Genes and Genomes) [18] by assigning KO (KEGG ontology) through BlastKOALA [19]. Gene category was also retrieved from KEGG.

\section{Pan-Genome analysis}

The web platform OrthoVenn2 [20] was used to identify orthologous gene clusters. OrthoVenn2 uses a modified version of the heuristic approach named OrthoMCL [21] to identify ortholog groups. An E-value cut off of $1 e^{-5}$ was used for all-to-all protein similarity comparisons. An inflation value of 1.5 was used for the generation of orthologous clusters using the Markov Cluster Algorithm [22]. A Venn diagram in $\mathrm{R}$ was used to construct the six-way Venn diagram of shared CDSs among C. ruddii strains.

\section{Results and Discussion}

\section{Genomic features of $C$. ruddii strains}

The main genomic features of six C. ruddii strains are summarized in Table 1. A circular genome map for each genome was constructed by using the BLAST Ring Image Generator. A visual inspection of the circular alignment of the genomes of $C$. ruddii stains revealed a relatively high sequence similarity (Fig. 1). A brief description of genomes of six $C$. ruddii strains from different host has been reported [13, 14, 23-25] but depth intra-species comparison of $C$. ruddii strains have not yet been done. All six C. ruddi strains contained a single circular chromosome and no plasmid was found. The genomes of $C$. ruddii strains are highly reduced and very similar in size from $157,543 \mathrm{bp}$ to $174,018 \mathrm{bp}$ with GC content varied from $13.98 \%$ to $17.63 \%$. Genome comparison showed that HT strain is reduced most in size (157,543 bp) and has smaller number of total genes. Contrarily, the YCCR strain has the larger genome content of $174,018 \mathrm{bp}$ with comparatively higher GC content (17.63\%) but lowest coding density of gene. The CE strain has the lowest GC content (13.98\%) among six strains. The genome size of BT strain is 174,004 bp but the coding density is much higher compared with other five strains. In general, the number of genes among six C. ruddii strains varied from 211 to 227 . Although the strains are isolated from different psyllids host species, they shared a high identity. All six C. ruddii strains contained three rRNA genes and four C. ruddii strains (BC, HT, PV and CE) has 28 tRNAs whereas each of $C$. ruddii strains BT and YCCR has 22 tRNAs. So, the overall genome size, number of genes and GC contents of $C$.

Table 1. General genomic features of six $C$. ruddii genomes.

\begin{tabular}{lcccccc}
\hline \multicolumn{1}{c}{ Strain name } & $\mathrm{BC}$ & $\mathrm{BT}$ & $\mathrm{YCCR}$ & $\mathrm{HT}$ & $\mathrm{PV}$ & $\mathrm{CE}$ \\
\hline Accession number & $\mathrm{CP} 019943.1$ & $\mathrm{CP} 024798.1$ & $\mathrm{CP} 012411.1$ & $\mathrm{CP003544.1}$ & $\mathrm{AP009180.1}$ & CP003541.1 \\
Genome size (bp) & 173,802 & 174,004 & 174,018 & 157,543 & 159,662 & 162,589 \\
Plasmid & 0 & 0 & 0 & 0 & 0 & 0 \\
Chromosome size (bp) & 173,802 & 174,004 & 174,018 & 157,543 & 159,662 & 162,589 \\
G+C content (\%) & 14.81 & 14.61 & 17.63 & 14.55 & 16.56 & 13.98 \\
Total number of genes & 227 & 224 & 224 & 211 & 213 & 221 \\
Total CDS & 196 & 199 & 168 & 180 & 182 & 190 \\
rRNA & 3 & 3 & 3 & 3 & 3 & 3 \\
tRNA & 28 & 22 & 22 & 28 & 28 & 28 \\
\hline
\end{tabular}




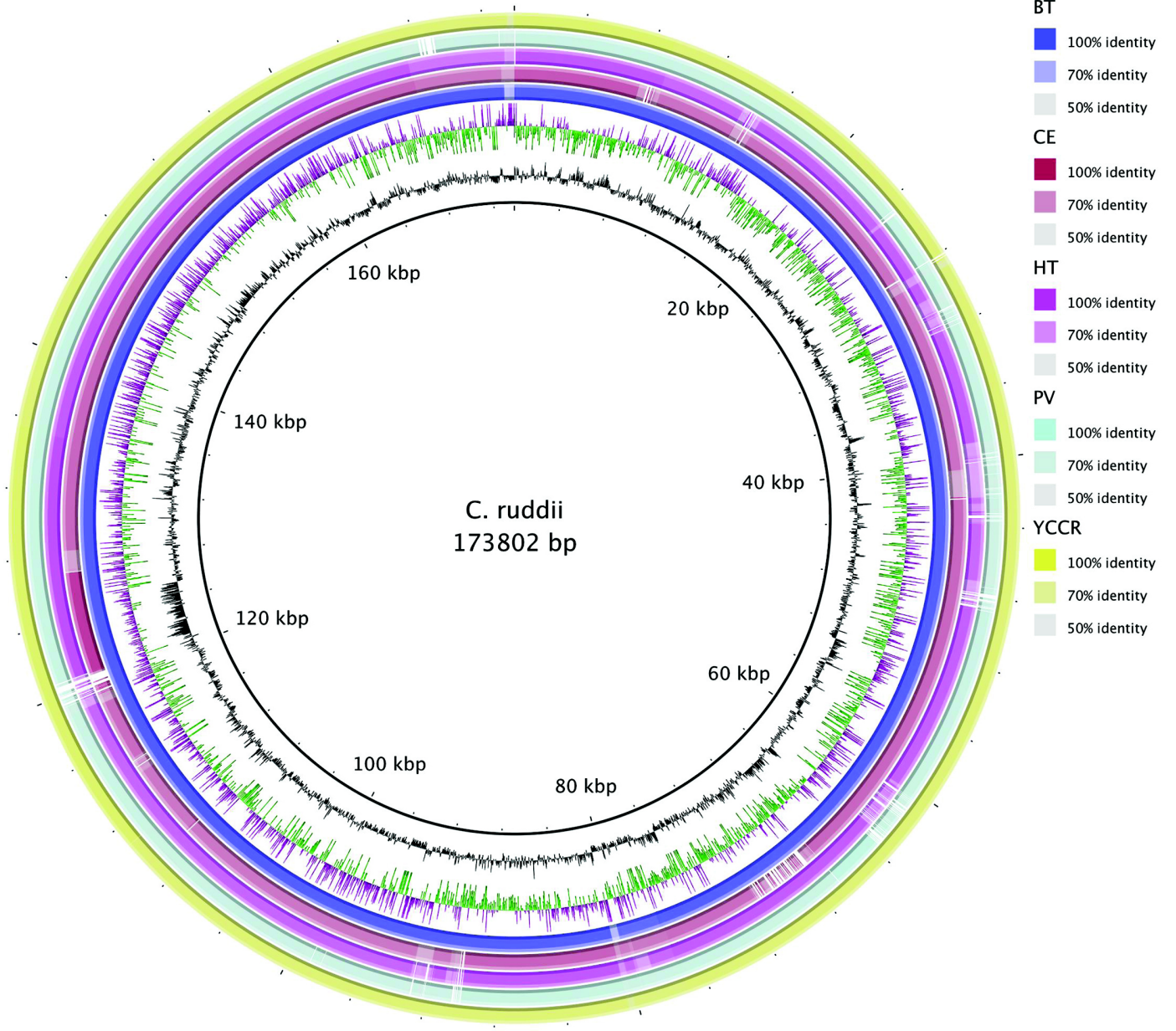

GC Content

Fig. 1. Comparative circular genome visualization of six C. ruddii strains. The BLASTN-based ring image of sequences was generated by BLAST Ring Image Generator (BRIG). Solid colour reflects $100 \%$ nt identity, fainter colours indicate between $50 \%$ and $80 \%$ nt identity and grey features indicate $<50 \%$ identity. From inside to outside: Ring 1: GC content, Ring 2: GC Skew, Ring 3: BLAST comparison with strain BT, Ring 4: BLAST comparison with strain CE, Ring 5: BLAST comparison with strain HT, Ring 6: BLAST comparison with strain PV, Ring 7: BLAST comparison with strain YCCR.

ruddii strains reduce following to the intracellular bacteria during specialization to the intracellular host lifestyle indicating a continuous selective pressure for minimal genome. The possible explanations for the genome reduction are due to the intracellular habitats that limit the gene acquisition capacity by lateral gene transfer [2629] and also the gene loss with increased adaption to the host [30, 31]. Due to genome reduction and AT-richness, the primary endosymbiont genomes spectacle a high degree of stability that is the outcome of convergent pat- tern of evolution.

\section{Phylogenetic analysis}

A neighbor-joining phylogenetic tree based on the whole genome proteomes of 30 bacterial strains was constructed using the CVTree3 tool (See Materials and Methods). The resulting NJ tree showed that all the $C$. ruddii strains formed a cluster of strong supported monophyletic group where the six $C$. ruddii strains are placed in same clade (Fig. 2 and Supplementary Table 


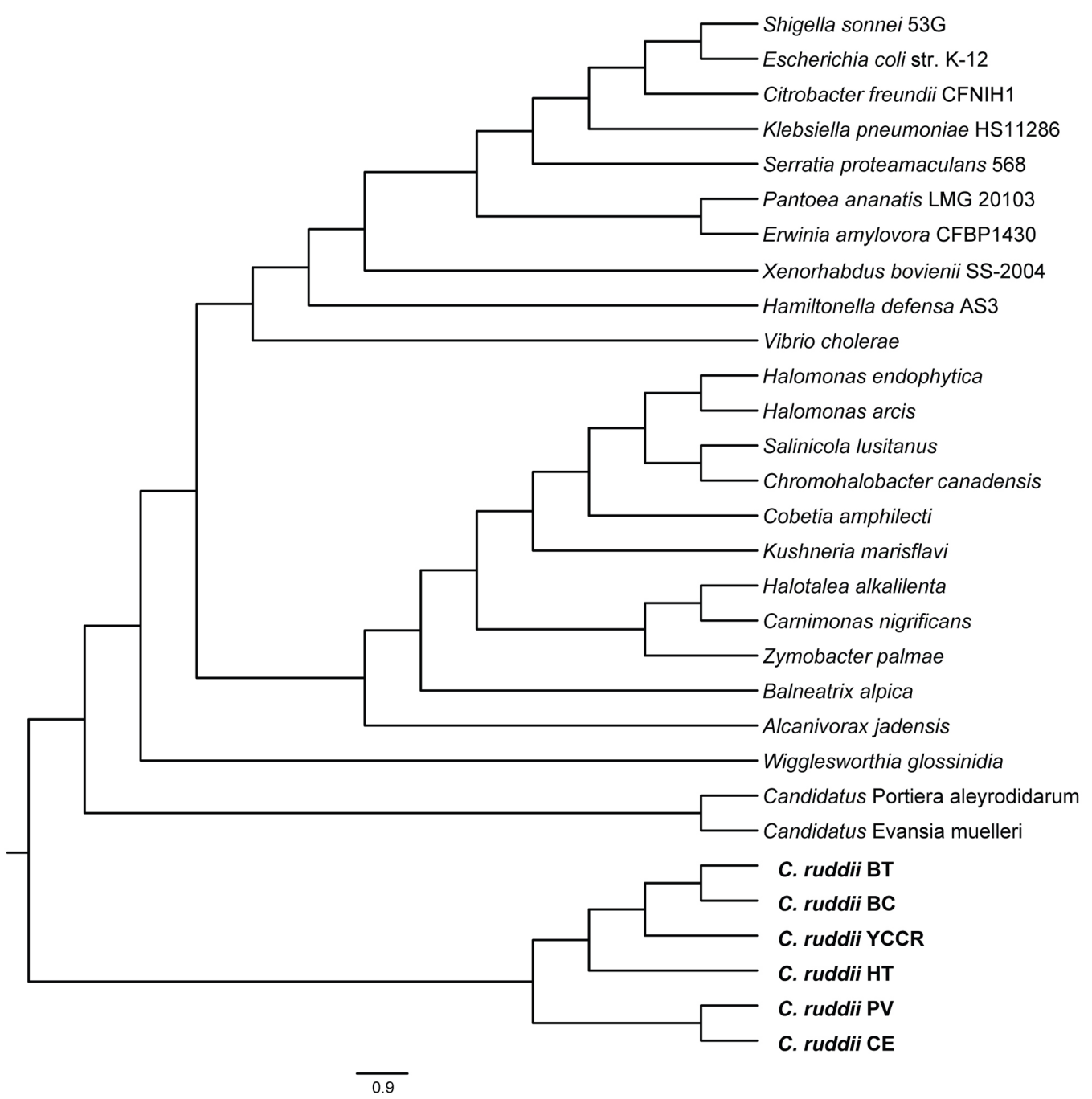

Fig. 2. Phylogenomics analysis of $\boldsymbol{C}$. ruddii strains. A neighbor-joining phylogenetic tree based on the whole genome proteomes of six C. ruddii strains from different psyllid host species and other closely related 24 bacterial strains (total 30 strains) from gamaproteobacteria class. This phylogenetic tree showed that the psyllid endosymbionts formed a cluster of strong supported monophyletic group where the six C. ruddii strains are placed in the same clade.

1). The reliable placement of $C$. ruddii genome in gamaproteobacteria is still in confusion due to AT richness and extreme amino acid bias, although the other insect symbiont with small AT rich genomes consisting of Candidatus Buchnera, Candidatus Baumannia, Candidatus Blochmannia, and Wigglesworthia appeared as a single clade in gamaproteobacteria [32]. Within a single monophyletic clade, the six $C$. ruddii strains are organized into different sub-clades, which also support the consistent division in host psyllid phylogeny [11]. The phylogenies of endosymbionts and their hosts are generally congruent evidenced by their long-term evolu- tion [33-35].

\section{Pan genome reconstruction}

Comparative analysis of presence/absence of gene families among the six $C$. ruddii strains reveals that the pangenome contains a total of 204 genes. The core shared by all the strains accounts for 133 gene families (orthogroups) (Fig. 3). No orthogroups were unique to these six strains. Whereas, some orthogroups were shared by two or more strains. For instance, 12, 10, 17 and 29 orthogroups were shared by two, three, four and five strains, respectively. The orthologous groups specific 


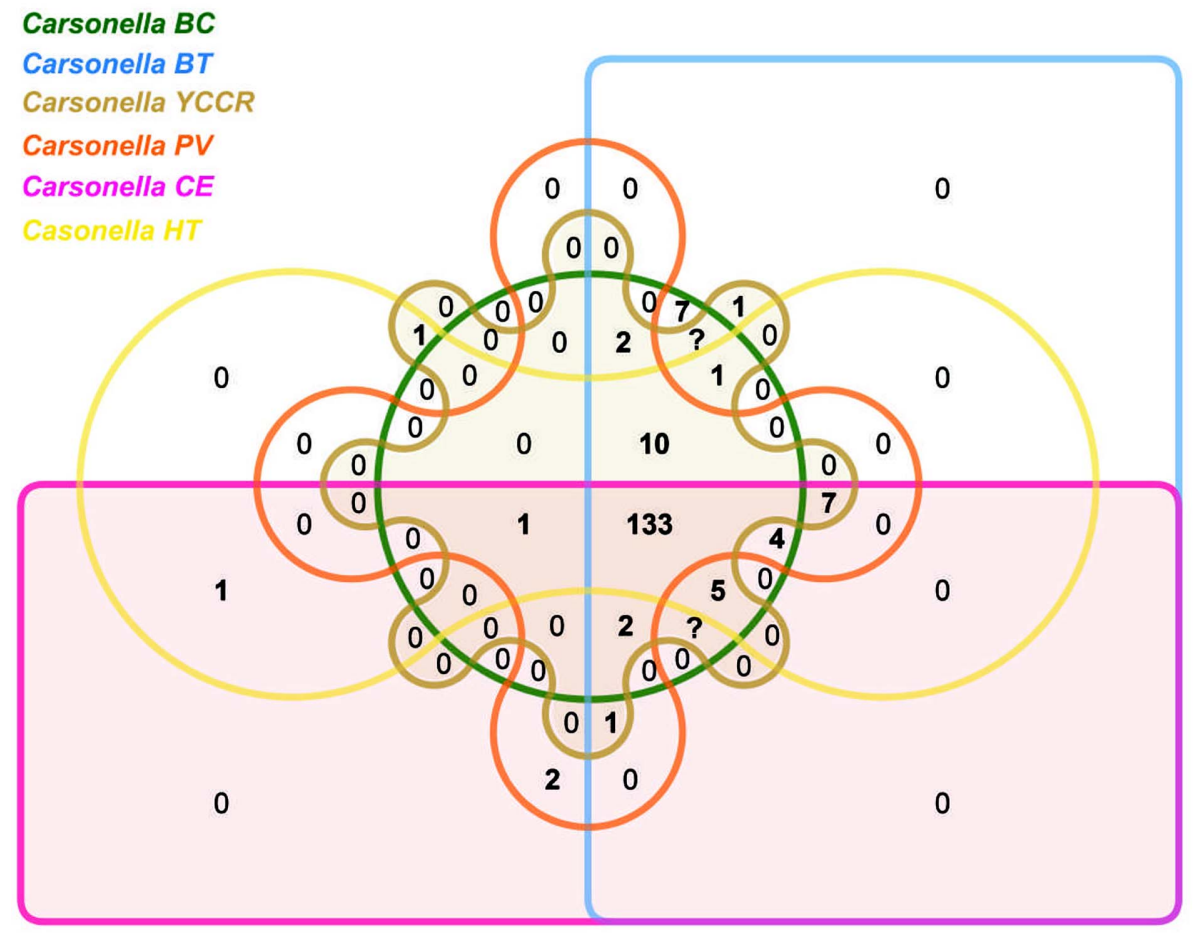

Fig. 3. The Venn diagram depicting shared and distinct protein clusters of six C. ruddii strains. Coloured circle/box represent six different strains. The number of clusters is provided in the intersections. Among these clusters, 133 are common to all strains, whereas no orthogroups were unique to these six strains. Some orthogroups were shared by two or more strains. The web platform OrthoVenn2 [20] was used to identify orthologous gene clusters.

to two C. ruddii strains were hypothetical protein except one was ribosomal protein. Whereas, three C. ruddii strains specific genes include tryptophan biosynthesis genes which also observed in metabolic reconstruction (detailed in biosynthetic pathway analysis). These results support the hypothesis that the reduced symbiotic genomes tend to retain core genes dedicated to maintaining the symbiotic relationship and the central

Table 2. Gene number distribution for different functional category analysis in six C. ruddii strains.

\begin{tabular}{lllllll}
\hline \multicolumn{1}{c}{ Strain } & BC & BT & YCCR & HT & PV & CE \\
\hline Genetic Information Processing & 83 & 84 & 58 & 84 & 88 & 84 \\
Amino acid metabolism & 61 & 64 & 52 & 45 & 47 & 51 \\
Cellular Processes & 14 & 14 & 12 & 12 & 12 & 13 \\
Energy metabolism & 13 & 13 & 12 & 14 & 14 & 14 \\
Carbohydrate metabolism & 12 & 13 & 11 & 13 & 13 & 14 \\
Nucleotide metabolism & 11 & 10 & 9 & 9 & 9 & 8 \\
Metabolism of cofactors and vitamins & 9 & 9 & 9 & 8 & 5 & 8 \\
Secondary metabolites biosynthesis & 7 & 8 & 4 & 5 & 5 & 7 \\
Human Diseases & 8 & 8 & 7 & 7 & 7 & 8 \\
Organismal Systems & 5 & 5 & 4 & 0 & 0 & 5 \\
Unclassified & 5 & 5 & 0 & 5 & 5 & 0 \\
Metabolism of other amino acids & 0 & 0 & 5 & 5 & 5 & 5
\end{tabular}




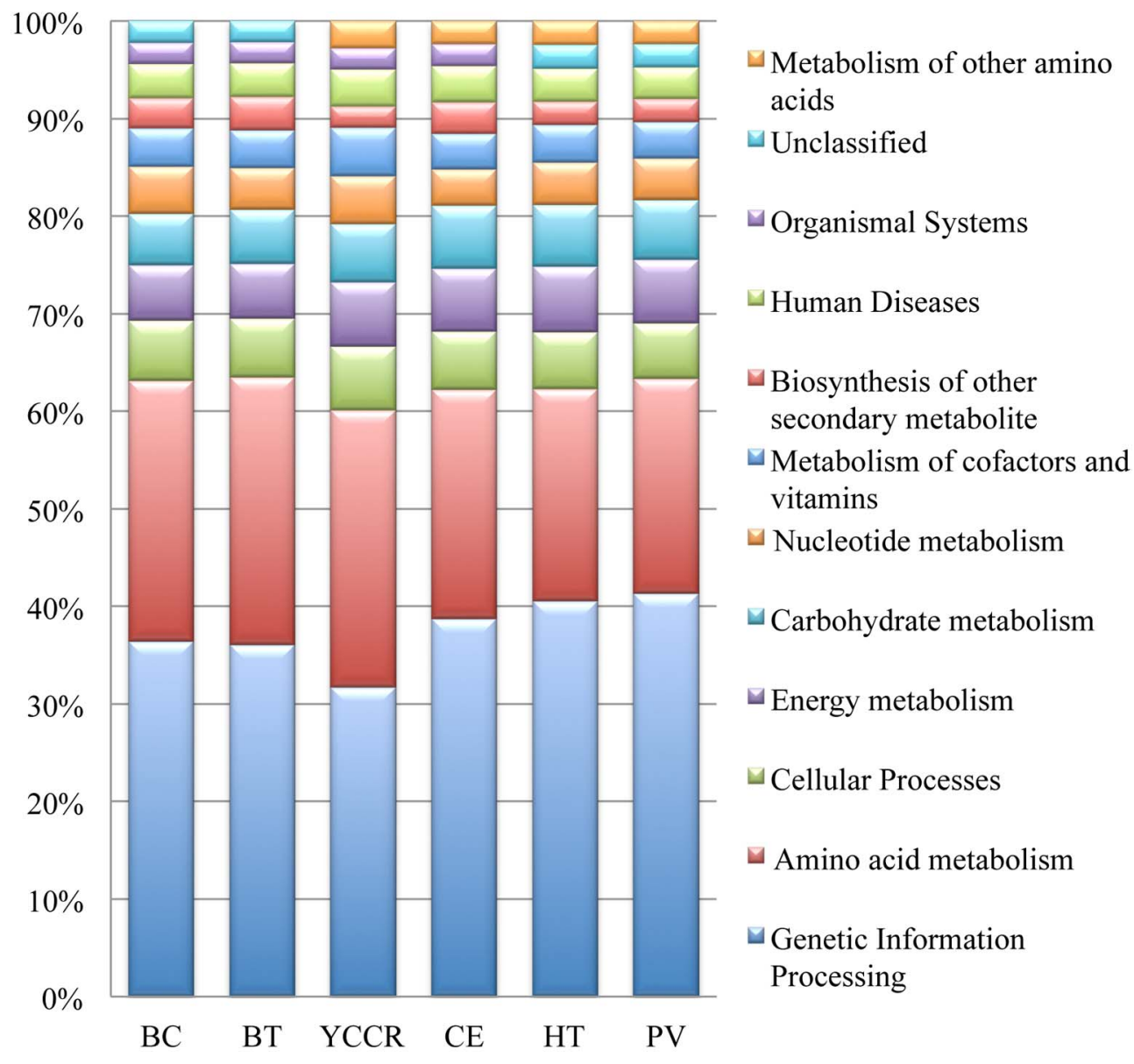

Fig. 4. Distribution of genes in functional categories obtained from KEGG for six C. ruddii strains. The functional categories (amino acid metabolism, cellular process, energy metabolism, carbohydrate metabolism, nucleotide metabolism, metabolism of cofactors and vitamins, biosynthesis of other secondary metabolite, human diseases, organismal systems, unclassified and metabolism of other amino acids) were assigned by KEGG. Percentages indicate the sum of genes in each functional category (indicated by color).

informational machinery [36]. It also suggested that a small number of species-specific genes are present in reduced symbiotic genome, which may play important roles in the specific biology/life styles of each symbiont.

\section{Functional category analysis}

The overall information about number of genes involved in several functional categories obtained from KEGG database are listed in (Table 2) which reveals that the most represented functional category in the endosymbiont C. ruddii genomes consists of genes involved in genetic information processing, accounting for about $40 \%$ (Fig. 4). The second most represented functional category is amino acid metabolism involves about $25 \%$ of all genes. Almost all the six strains have same percentage of gene involved in several categories including cellular process (7\%), energy metabolism (7\%), carbohydrate metabolism (7\%), nucleotide metabolism (5\%), metabolism of cofactors and vitamin biosynthesis (5\%) and others (4\%). The comparative analysis clearly shows that gene complement is not sufficient to replicate, transcribe and synthesize proteins. It is already proved for $C$. ruddii $\mathrm{PV}$ strain that most of the genes for DNA replication, transcription and translation are absent, the transcription machinery is limited and the translation machinery is highly reduced. Extensive functional analysis with six $C$. ruddii strains revealed the same pattern of genes like the PV strain. As all six $C$. ruddii genomes lacks most of the genes for DNA replication, transcription and translation so the biological 


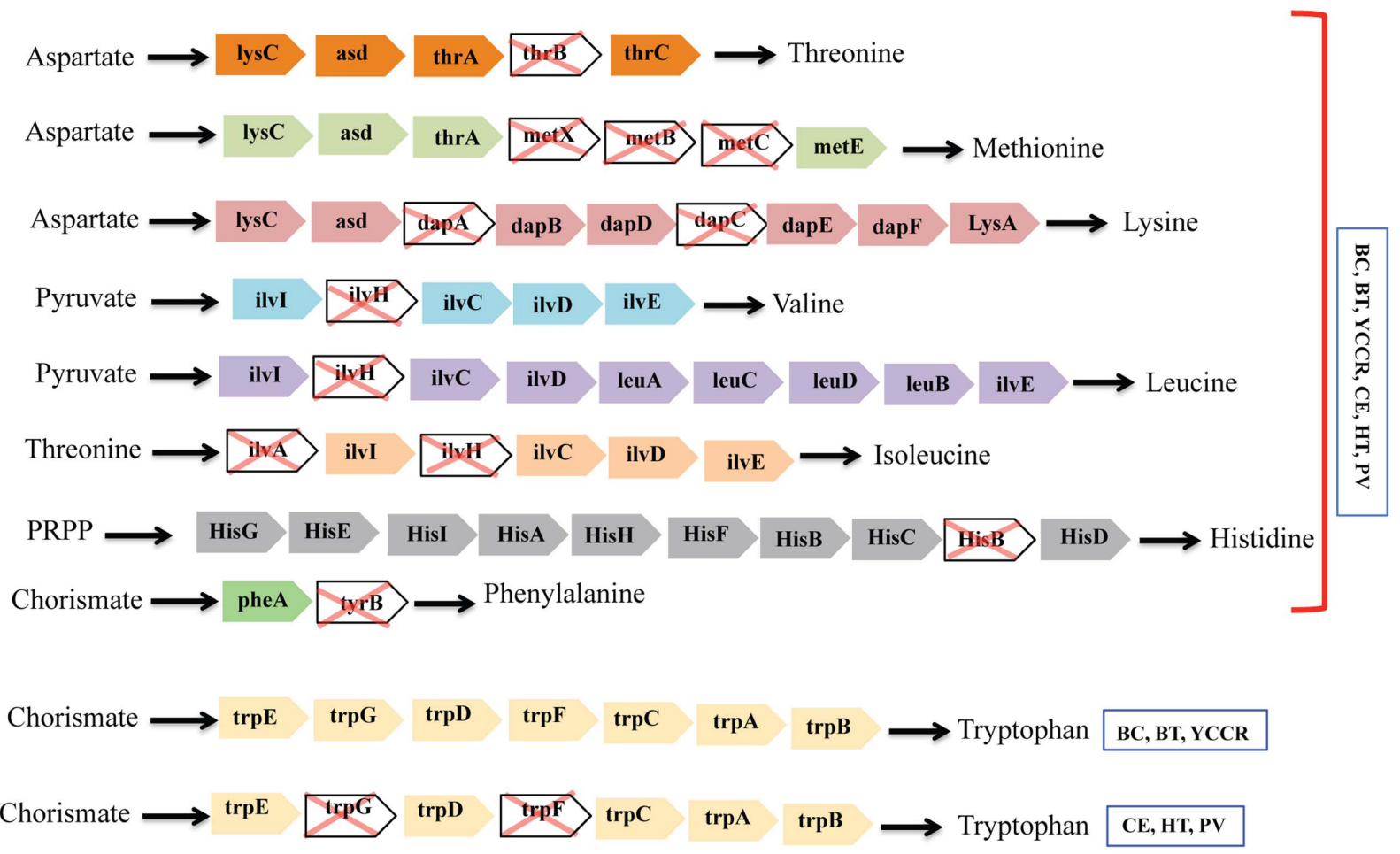

Fig. 5. Biosynthesis pathways for nine essential amino acids in six C. ruddii strains. Gene names are indicated in colored rectangles. White rectangles with red $\times$ indicated missing genes. All six strains have similar set of genes for eight essential amino acids biosynthesis, which is indicated by right bracket. Only for tryptophan biosynthesis, three C. ruddii strains (BC, BT, YCCCR) have complete set of genes and another three C. ruddii strains ( $C E, H T, P V)$ comprise two missing genes, which is indicated by green right bracket.

entity of $C$. ruddii genomes might be dependent on external source for these functions.

\section{Biosynthetic pathway analysis}

Despite the drastic genome reduction, the $C$. ruddii genomes retained many genes responsible for several cellular processes as well as amino acids biosynthesis or metabolism. We present a detailed biosynthetic pathway of the genes retained in $C$. ruddii genomes which is important to know the physiological role of this genome as a primary endosymbiont. Complete set of genes for eight essential amino acids like lysine, methionine, threonine, phenylalanine, valine isoleucine, leucine and histidine, are absent in all six C. ruddii strains whereas all genes for tryptophan biosynthesis are present in three C. ruddii strains (BC, BT, YCCR) but some genes are absent in another three $C$. ruddii strains (CE, HT, PV) (Fig. 5). Whereas, genes for most nonessential amino acids (glutamate, serine, alanine, asparagine, glutamine and proline) are absent except cysteine. Carsonella rud- dii seemed don't have the ability to produce endogenously important precursors such as erythrose-4phosphate, aspartate and pyruvate.

The expected result arouse from this analysis revealed that most of the essential amino acids biosynthetic pathways are completely or partially lost for all six C. ruddii strains. The psyllid mainly feed the plant phloem sap which is rich in sugar but deficit in nitrogenated compounds, especially essential amino acids [37].

It is probable that the endosymbionts of insects that feed on plant phloem sap mainly provide the host with missing essential nutrients like Buchnera aphidicola of aphids and Blochmannia spp. of ants provide essential amino acids and vitamins that are deficient in the host diet [13, 23, 38]. But unlikely, due to the limited number of amino acid biosynthesis pathway genes, C. ruddii stains are unable to supply the essential amino acids to the host. The total gene content of $C$. ruddii strains are almost half of the genes identified in B. aphidicola [38] and such small number of genes raise a question to full 
fill the functional characteristic of $C$. ruddii as a living cell. The extensive small genome size of $C$. ruddii strains are not compatible to support all the essential genes to be considerate as a mutualistic endosymbiont even more as a living organism. In some insect-bacteria association, the essential endosymbioyic function has lost for primary endosymbiont and the missing function might compensate from other secondary endosymbiont, which plays the vital role to complete deficiency of the insect diet [23, 39]. Aside from the ability to supply the essential amino acids from primary endosymbiont, it is important that the psyllid may rely on the secondary endosymbiont for maintaining their life cycle. It is therefore possible that $C$. ruddii is being driven to their extinction and replacement by new symbionts as it has already been proposed in other insects [15].

\section{Conclusion}

We first performed the depth intra-species comparison of six C. ruddii strains and showed C. ruddii is not compatible with its consideration as a mutualistic endosymbiont. The extensive small genome size of $C$. ruddii strains raise a question to full fill the functional characteristic of $C$. ruddii as a living cell. Although $C$. ruddii is defined as a psyllid primary symbiont, the genes for some essential amino acids pathway are absent. So, it is important that the psyllid may rely on the secondary endosymbiont for maintaining their life cycle. It is therefore possible that $C$. ruddii is being driven to their extinction and replacement by new symbionts.

\section{Acknowledgments}

We would like to thank Genetic Engineering and Biotechnology Department, Shahjalal University of Science and Technology, Sylhet, Bangladesh for providing facilities for the project.

\section{Conflict of Interest}

The authors have no financial conflicts of interest to declare.

\section{References}

1. Gray SM, Banerjee N. 1999. Mechanisms of arthropod transmission of plant and animal viruses. Microbiol. Mol. Biol. Rev. MMBR. 63: 128-148.
2. Blackman RL, Eastop VF. 1984. Aphids on the World's Crops: An Identification and Information Guide, pp. 476. 1st Ed. John Wiley Sons, London.

3. Sylvester ES. 1985. Multiple acquisition of viruses and vectordependent prokaryotes: consequences on transmission. Ann. Rev. Entomol. 30: 71-88.

4. Brown JK, Rehman M, Rogan D, Martin RR, Idris AM. 2010. First report of "Candidatus Liberibacter psyllaurous" (synonym "Ca. L. solanacearum") associated with 'Tomato Vein-Greening' and 'Tomato Psyllid Yellows' diseases in commercial greenhouses in Arizona. Plant Dis. 94: 376.

5. Munyaneza J, Buchman J, Upton J, Goolsby J, Crosslin J, Bester G, et al. 2008. Impact of different potato psyllid populations on zebra chip disease incidence, severity, and potato yield. Subtropical Plant Sci. 60: 27-37.

6. Dan H, Ikeda N, Fujikami M, Nakabachi A. 2017. Behavior of bacteriome symbionts during transovarial transmission and development of the Asian citrus psyllid. PLoS One. 12: e0189779.

7. Baumann P, Moran NA, Baumann L. 2006. Bacteriocyte-Associated Endosymbionts of Insects, pp. 403-438. In Dworkin M, Falkow S, Rosenberg E, Schleifer K-HandStackebrandt E (eds.), Ed. Springer New York, New York, NY.

8. Baumann P, Baumann L, Lai CY, Rouhbakhsh D, Moran NA, Clark MA. 1995. Genetics, physiology, and evolutionary relationships of the genus Buchnera: intracellular symbionts of aphids. Ann. Rev. Microbiol. 49: 55-94.

9. Thao ML, Clark MA, Baumann L, Brennan EB, Moran NA, Baumann P. 2000. Secondary endosymbionts of psyllids have been acquired multiple times. Curr. Microbiol. 41: 300-304.

10. Engel P, Moran NA. 2013. The gut microbiota of insects - diversity in structure and function. FEMS Microbiol. Rev. 37: 699-735.

11. Thao ML, Moran NA, Abbot P, Brennan EB, Burckhardt DH, Baumann P. 2000. Cospeciation of psyllids and their primary prokaryotic endosymbionts. Appl. Environ. Microbiol. 66: 2898-2905.

12. Sacchi L, Genchi M, Clementi E, Negri I, Alma A, Ohler S, et al. 2010. Bacteriocyte-like cells harbour Wolbachia in the ovary of Drosophila melanogaster (Insecta, Diptera) and Zyginidia pullula (Insecta, Hemiptera). Tissue Cell 42: 328-333.

13. Nakabachi $A$, Yamashita $A$, Toh $H$, Ishikawa $H$, Dunbar HE, Moran NA, et al. 2006. The 160-kilobase genome of the bacterial endosymbiont Carsonella. Science 314: 267.

14. Sloan DB, Moran NA. 2012. Genome reduction and co-evolution between the primary and secondary bacterial symbionts of psyllids. Mol. Biol. Evol. 29: 3781-3792.

15. Tamames J, Gil R, Latorre A, Peretó J, Silva FJ, Moya A. 2007. The frontier between cell and organelle: genome analysis of Candidatus Carsonella ruddii. BMC Evol. Biol. 7: 181.

16. Alikhan NF, Petty NK, Ben Zakour NL, Beatson SA. 2011. BLAST Ring Image Generator (BRIG): simple prokaryote genome comparisons. BMC Genomics 12: 402.

17. Zuo G, Hao B. 2015. CVTree3 web server for whole-genome-based and alignment-free prokaryotic phylogeny and taxonomy. Genomics Proteomics Bioinformatics 13: 321-331. 
18. Ogata H, Goto S, Sato K, Fujibuchi W, Bono H, Kanehisa M. 1999. KEGG: Kyoto encyclopedia of genes and genomes. Nucleic Acids Res. 27: 29-34.

19. Kanehisa M, Sato Y, Morishima K. 2016. BlastKOALA and GhostKOALA: KEGG Tools for functional characterization of genome and metagenome sequences. J. Mol. Biol. 428: 726-731.

20. Xu L, Dong Z, Fang L, Luo Y, Wei Z, Guo H, et al. 2019. OrthoVenn2: a web server for whole-genome comparison and annotation of orthologous clusters across multiple species. Nucleic Acids Res. 47: W52-w58.

21. Li L, Stoeckert CJ, Jr., Roos DS. 2003. OrthoMCL: identification of ortholog groups for eukaryotic genomes. Genome Res. 13: 21782189.

22. Enright AJ, Van Dongen S, Ouzounis CA. 2002. An efficient algorithm for large-scale detection of protein families. Nucleic Acids Res. 30: 1575-1584.

23. Wu F, Deng X, Liang G, Huang J, Cen Y, Chen J. 2015. Wholegenome sequence of "Candidatus Profftella armatura" from diaphorina citri in Guangdong, China. Genome Announc. 3: e0128201215

24. Riley AB, Kim D, Hansen AK. 2017. Genome sequence of "Candidatus Carsonella ruddii" strain $\mathrm{BC}$, a nutritional endosymbiont of bactericera cockerelli. Genome Announc. 5: e00236-17.

25. Katsir L, Zhepu R, Piasezky A, Jiang J, Sela N, Freilich S, et al. 2018. Genome sequence of "Candidatus Carsonella ruddii" strain BT from the psyllid bactericera trigonica. Genome Announc. 6: e01466-01417.

26. Wernegreen JJ. 2005. For better or worse: genomic consequences of intracellular mutualism and parasitism. Curr. Opin. Genet. Dev. 15: 572-583.

27. Tamas I, Klasson L, Canback B, Naslund AK, Eriksson AS, Wernegreen JJ, et al. 2002. 50 million years of genomic stasis in endosymbiotic bacteria. Science (New York, N.Y.). 296: 2376-2379.

28. Moran NA, Plague GR. 2004. Genomic changes following host restriction in bacteria. Curr. Opin. Genet. Dev. 14: 627-633.

29. Audic S, Robert C, Campagna B, Parinello H, Claverie JM, Raoult D, et al. 2007. Genome analysis of Minibacterium massiliensis highlights the convergent evolution of water-living bacteria. PLoS Genet. 3: e138.

30. Moran NA. 2002. Microbial minimalism: genome reduction in bacterial pathogens. Cell 108: 583-586.

31. Darby AC, Cho NH, Fuxelius HH, Westberg J, Andersson SG. 2007. Intracellular pathogens go extreme: genome evolution in the Rickettsiales. Trends Genet. 23: 511-520.

32. Williams KP, Gillespie JJ, Sobral BWS, Nordberg EK, Snyder EE, Shallom JM, et al. 2010. Phylogeny of Gammaproteobacteria. J. Bacteriol. 192: 2305.

33. Lo N, Bandi C, Watanabe H, Nalepa C, Beninati T. 2003. Evidence for cocladogenesis between diverse dictyopteran lineages and their intracellular endosymbionts. Mol. Biol. Evol. 20: 907-913.

34. Conord C, Despres L, Vallier A, Balmand S, Miquel C, Zundel S, et al. 2008. Long-term evolutionary stability of bacterial endosymbiosis in curculionoidea: additional evidence of symbiont replacement in the dryophthoridae family. Mol. Biol. Evol. 25: 859-868.

35. Chaumot A, Da Lage JL, Maestro O, Martin D, Iwema T, Brunet F, et al. 2012. Molecular adaptation and resilience of the insect's nuclear receptor USP. BMC Evol. Biol. 12: 199.

36. Moran NA, Bennett GM. 2014. The tiniest tiny genomes. Ann. Rev. Microbiol. 68: 195-215.

37. Baumann P. 2005. Biology bacteriocyte-associated endosymbionts of plant sap-sucking insects. Ann. Rev. Microbiol. 59: 155189.

38. Gil R, Silva FJ, Peretó J, Moya A. 2004. Determination of the core of a minimal bacterial gene set. Microbiol. Mol. Biol. Rev. 68: 518 .

39. Perez-Brocal V, Gil R, Ramos S, Lamelas A, Postigo M, Michelena $\mathrm{JM}$, et al. 2006. A small microbial genome: the end of a long symbiotic relationship? Science 314: 312-313. 